\title{
MANIFOLDS OF THE HOMOTOPY TYPE OF (NON-LIE) GROUPS
}

\author{
BY JAMES D. STASHEFF
}

Communicated by Edgar Brown, February 17, 1969

Hilbert's Fif th Problem implies that a topological group which is topologically a finite dimensional manifold is a Lie group. Until quite recently, the only topological groups of the homotopy type of compact manifolds known were Lie groups. In 1963 Slifker exhibited a topological group of the homotopy type of $S^{3}$ yet not multiplicatively equivalent to SU(1). In 1968, Hilton and Roitberg announced the discovery of a 10-dimensional manifold $M_{7}^{10}$ which admits a multiplication yet is not of the homotopy type of a Lie group. In fact, they showed $M_{7}^{10} \times S^{3}=\mathrm{Sp}(2) \times S^{3}$. They left open the question: Does $M_{7}^{10}$ admit a homotopy associative multiplication, a necessary condition for $M^{10}$ to be of the homotopy type of a topological group? We answer the question affirmatively; thus a homotopy version of Hilbert's Fifth Problem is false.

THEOREM 1. There is a topological group $G$ of the homotopy type of a compact manifold $M^{10}$ (the 3-sphere bundle over $S^{7}$ described by Hilton and Roitberg) which is not of the homotopy type of any Lie group.

More precisely we show the following

THEOREM 2. Let $S^{3} \rightarrow M_{n}^{10} \rightarrow S^{7}$ be the principal $S^{3}$-bundle classified by $n \omega \in \pi_{6}\left(S^{3}\right), n \in Z_{12}$, $\omega$ chosen as a generator such that the corresponding $M_{1}^{20}$ is $\mathrm{Sp}(2)$.

$M_{n}^{10}$ is of the homotopy type of a Lie group if and only if $n \equiv \pm 1$ (12).

$M_{n}^{10}$ is of the homotopy type of a topological group if $n \equiv \pm 1, \pm 5$ (12).

$M_{n}^{10}$ admits a multiplication if $n \neq 2(4)$.

The first part results from the classification of such bundles up to homotopy type and the classification of Lie groups. The case $n \equiv-1$ is realized by $\overline{\mathrm{Sp}}(2)$, the opposite symplectic group, which has the same underlying space as Sp (2) but the opposite order of multiplication.

The remainder of the theorem is proved using a new technique of Zabrodsky's called "mixing homotopy types" [2].

Let $P$ be the set of primes and $P=P_{1} \cup P_{2}$, a decomposition into disjoint subsets. Let $\mathcal{e} P_{1}$ denote the class of abelian groups of orders not divisible by primes in $\boldsymbol{P}_{2}$ and let $\mathbf{C} P_{2}$ denote the class of abelian groups not divisible by primes in $P_{1}$.

Let $X, X_{0}$ be simply connected $\mathrm{CW}$-complexes. 
THEOREM 3. Let $f: X \rightarrow X_{0}$ be a rational homotopy equivalence. There exists a space $X\left(P_{1}\right)$ and a factorization $X \stackrel{f_{1}}{\rightarrow} X\left(P_{1}\right) \stackrel{f_{2}}{\rightarrow} X_{0}$ of $f$ such that the fibre of $f_{i}$ has homotopy groups belonging to $\mathrm{eP}_{i}$.

If $X, X_{0}$ are $H$-spaces and $f$ an $H$-map, then $X\left(P_{1}\right)$ is an $H$-space and $f_{2}, f_{1}$ are $H$-maps.

Theorem 4. Let $X_{i}$ be simply connected $\mathrm{CW}$-complexes for $i=0,1,2$. Let $f_{i}: X_{i} \rightarrow X_{0}$ be a rational homology equivalence.

There exists a space $X$ and maps $g_{i}: X \rightarrow X_{i}\left(P_{i}\right)$ such that the fibre has homotopy groups belonging to $\mathcal{e} P_{i \pm 1}$. If the "ingredients" $X_{i}, f_{i}$ are $H$-spaces and $H$-maps, then $X$ is an $H$-space and the maps $g_{i}$ are H-maps.

If the ingredients are topological groups and homomorphisms, $X$ has the homotopy type of a topological group.

Theorem 3 can be proved by constructing a modified MoorePostnikov system for $f$ in which the primary components of the homotopy groups $\pi_{i}\left(X_{0}, X\right)$ are put in first for $p \in P_{1}$ and then for $p \in P_{2}$. More simply, since $f$ is a rational equivalence, its fibre $F$ has the homotopy type of a product $\prod_{p \in P} F_{p}$ where $F_{p}$ has $p$-primary homotopy only. $X\left(P_{1}\right)$ can be thought of as the subfibration of $X$ in which the fibre is cut down to $\prod_{p \in P_{1}} F_{p}$.

To obtain the $H$-conditions, the following specific details are helpful. $X\left(P_{1}\right)$ can be constructed by a succession of principal $K\left(Z_{p}, n\right)$-fibrations, $p \in P_{1}$ induced by cohomology classes in the kernel of the cohomology morphism $\bmod p$.

Lemma. Let $f: X \rightarrow X^{1}$ be an $H$-map. If $f^{*}: H^{i}\left(X^{1}, Z_{p}\right) \rightarrow H^{i}\left(X, Z_{p}\right)$ is an isomorphism for $i<n-1$, monomorphism for $i=n-1$, and $\alpha \in \operatorname{Ker} f^{*} \cap H^{n}\left(X^{1}, Z_{p}\right)$ then $\alpha$ is represented by an $H$-map. The fibration $Y$ induced over $X^{1}$ by $\alpha$ is therefore an $H$-space such that $f$ can be lifted to an $H$-map $X \rightarrow Y$.

That $\alpha$ is represented by an $H$-map follows from the fact that $f^{*} \alpha=0$ is represented by an $H$-map and the obstructions lie where $(f \times f)^{*}$ is an isomorphism. The vanishing of these obstructions can thus be achieved in terms of chains whose images in $X^{2}$ are specified so the lifting of $f$ is immediate.

Elements in the cokernel of the cohomology morphism are added by trivial principal $\left(Z_{p^{r}}, n\right)$-fibrations.

Theorem 4 is proved by taking $X$ to be the fibre product (pull back) of $f_{i}: X_{i}\left(P_{i}\right) \rightarrow X_{0}$ : 


$$
\begin{array}{cc}
X & \rightarrow X_{1}\left(P_{1}\right) \\
\downarrow & \downarrow \\
X_{2}\left(P_{2}\right) & \rightarrow \quad X_{0}
\end{array}
$$

If the ingredients are topological groups and homomorphisms, Browder observed the construction can also be carried out in terms of $B X_{i}$ to produce a classifying space $Y$. We then have $X$ of the homotopy type of $\Omega Y$ and hence of the homotopy type of a topological group by Milnor's constructions, if all spaces are countable CW.

Proposition. If $X_{1}, X_{2}$ are simply connected finite complexes, then $X$ has the homotopy type of a finite complex.

Proof. Since $H^{*}\left(X_{i} ; Q\right)$ is finite dimensional as a $Q$-vector space so is $H^{*}(X ; Q)$. Since $H^{*}\left(X_{i} ; Z_{p}\right)$ is finite dimensional for each $p, i=1,2$, so is $H^{*}\left(X ; Z_{p}\right)$. Moreover, the finite dimension has a common finite upper bound for $Q$ and all $p$ simultaneously (i.e. the maximum for $X_{1}, X_{2}$ ). Thus $X$ has the homotopy type of a finite complex, for example that obtained by a homology decomposition of $X$.

We are now ready for examples. Let $\overline{\mathrm{Sp}}(2)$ denote the "opposite symplectic group," i.e. the symplectic group obtained by multiplying quaternions in the opposite order. If $\omega \in \pi\left(S^{3}\right)$ is chosen as the generator which classifies $S^{3} \rightarrow \mathrm{Sp}(2) \rightarrow S^{7}$, then $\overline{\mathrm{Sp}}(2)$ is classified by $-\omega$. Recall that $\pi_{6}\left(S^{3}\right) \approx Z_{4}+Z_{3}$ with generators $\nu^{\prime}, \alpha[1]$. We have $\omega=\nu^{\prime}+\alpha$. If we mix the homotopy type of $X_{1}=\mathrm{Sp}(2)$ and $X_{2}=\overline{\mathrm{Sp}}(2)$ over $K(Z, 3) \times K(Z, 7)$ with $2 \in P_{2}$ and $3 \in P_{1}$, the resulting group is the Hilton-Roitberg example, for $-\nu^{\prime}+\alpha=7 \omega$. Interchanging $\mathrm{Sp}(2)$ and $\overline{\mathrm{Sp}}(2)$ gives a group classified as a bundle by $5 \omega$.

If we take $X_{1}=\mathrm{Sp}(2)$ and $X_{2}=S^{3} \times S^{7}$ with $2 \in P_{1}$ and $3 \in P_{2}$ then the bundle classified by $\nu^{\prime}=9 \omega$ is seen to admit a multiplication but not a homotopy associative one $\left[\rho^{1}: H^{3} \rightarrow H^{7}\right.$ is trivial which contradicts the nontriviality of cup cubes in the projective 3-spaces for $X]$. The same holds for $3 \omega$.

If we interchange the roles of $\mathrm{Sp}(2)$ and $S^{3} \times S^{7}$ then we see the bundles classified by $\pm 4 \omega= \pm \alpha$ admit multiplications. The multiplication may be homotopy associative, although $S^{7}(\{2\})$ admits no homotopy associative multiplication.

\section{REFERENCES}

1. H. Toda, Composition methods in homotopy groups of spheres, Ann. of Math. Studies, no. 49, Princeton Univ. Press, Princeton, N. J., 1962.

2. A. Zabrodsky, Homotopy associativity and finite $C W$ complexes, Mimeographed Notes, University of Illinois, Chicago Circle, Ill., 1968.

Princeton University, Princeton, New Jersey 08540

University of Notre Dame, Notre Dame, Indiana 46556 